compensations and responses to iron deficiency treatment between HHT molecular genotypes.

Results Serum ferritin was higher in ACVRL1 (median 31; IQR 17.5,75) than ENG (median 25; IQR 13,50.5; $p=0.006$ ) and SMAD4 (median 26; IQR 5,39.5; $p=0.03$ ) HHT/PAVM patients, as shown by Kruskal-Wallis and Dunn's post-test. Age and sex-adjusted linear regression analysis found that a $S M A D 4$ variant was predictive of a decrease in serum iron $(p<0.0005)$. Mean corpuscular volume was lower in SMAD4 (median 75; IQR 70,87) than ACVRL1 (median 90; IQR 86,93; $p<0.0001$ ) and ENG (median 89; IQR 84,93; $p<0.0001)$ patients. This was compensated for by higher red blood cell counts in SMAD4 (median 5.4; IQR 5,6) than ACVRL1 (median 4.7; IQR 4.2,5; $p<0.0001$ ) and ENG (median 4.8; IQR 4.3,5.1; $p<0.0001$ ) patients, so that ultimately, haemoglobin concentrations did not differ significantly between molecular genotypes $(p=0.39)$. Associations between molecular genotype and other iron deficiency complications, such as ischemic stroke and venous thromboembolism are under evaluation.

Conclusions SMAD4 HHT/PAVM patients had lower iron indices, more marked indicators of iron deficiency anaemia, and displayed evidence of different compensatory mechanisms to maintain haemoglobin concentration. We speculate that the role of SMAD4 as a hepcidin regulator may explain why SMAD4 patients have this unique phenotype. A randomisedcontrol trial prospectively assessing differing molecular genotypes' responses to iron treatment would help to further clarify relationships between iron deficiency and HHT molecular genotype.

\section{S67 WHOLE GENOME SEQUENCING OF PATIENTS WITH PULMONARY ARTERIOVENOUS MALFORMATIONS AND HEREDITARY HAEMORRHAGIC TELANGIECTASIA}

${ }^{1} \mathrm{KE}$ Joyce, ${ }^{2}$ West London Genomic Medicine Centre Consortium, ${ }^{3}$ Genomics England Research Consortium, ${ }^{3} \mathrm{~F}$ Boardman-Pretty, ${ }^{3} \mathrm{CL}$ Shovlin. ${ }^{1}$ Imperial College School of Medicine, London, UK; ${ }^{2}$ Imperial College Healthcare NHS Trust, London, UK; ${ }^{3}$ Genomics England, England, UK

\subsection{6/thorax-2021-BTSabstracts.73}

Background The personalisation of medicine to target genetic modifications may benefit patients. A well characterised complex disease with a large variation in phenotype and whole genome sequencing could be used to determine new genetic links to disease manifestations. Pulmonary arteriovenous malformations (AVMs) provide one such disease, especially for the patients with underlying hereditary haemorrhagic telangiectasia (HHT). Both diseases were specifically recruited to the 100,000 Genomes Project.

Methods Literature searches for relevant genes that could impact on the variable phenotypes were performed. The genes of interest were investigated within the Research Environment of the 100,000 Genomes Project using LabKey. For each variant, potential pathogenicity was assigned using general population allele frequencies (GnomAD v3.1.1) and Combined Annotation Dependent Depletion (CADD) scores. Anonymised, categorised data were exported through the Research Environment Airlock in order to integrate with detailed blood results and sub-phenotypes.

Results The 75 genes of interest were split into five groups by their activity. 56 of these genes $(75 \%)$ had variants within the participants. All variants were rare with allele frequencies less than 0.003. CADD scores ranged from 0-42 where scores above 15 are commonly considered to indicate likely deleteriousness. There was a difference in CADD scores between the gene categories (Kruskal Wallis $\mathrm{p}=0.0073$ ), and the categories and genes with a greater variant burden in the study cohort also had higher gene damage indices in the general population. There was no difference in variants or genes according to the HHT gene (most commonly ENG or ACVRL1). Examination in relation to patient phenotypes is ongoing.

Conclusions Patients with pulmonary AVMs and HHT commonly have rare variants in genes of potential relevance to their phenotype. Phenotypic associations are required to establish if this is by chance or of pathophysiological importance.

Acknowledgements This research was made possible through access to the data and findings generated by the 100,000 Genomes Project, managed by Genomics England Limited funded by the Department of Health and Social Care, the National Institute for Health Research (NIHR) and NHS England.

\section{Gazing through the crystal ball: predicting outcomes from COVID-19}

\section{S68 NATIONAL COVID POINT OF CARE LUNG ULTRASOUND EVALUATION (SOCIETY FOR ACUTE MEDICINE WITH THE INTENSIVE CARE SOCIETY)}

${ }^{1} \mathrm{~T}$ Knight, ${ }^{2} \mathrm{P}$ Parulekar, ${ }^{3} \mathrm{G}$ Rudge, ${ }^{4} \mathrm{~F}$ Lesser, ${ }^{4} \mathrm{M}$ Dachsel, ${ }^{5} \mathrm{~A}$ Aujayeb, ${ }^{1} \mathrm{D}$ Lasserson, ${ }^{4} \mathrm{~N}$ Smallwood. 'Sandwell and West Birmingham Hospitals NHS Trust, Birmingham, UK; ${ }^{2}$ East Kent Hospitals NHS Trust, East Kent, UK; ${ }^{3}$ Institute of Applied Health Research University of Birmingham, Birmingham, UK; ${ }^{4}$ Surrey and Sussex Healthcare NHS Trust, Redhill, UK; ${ }^{5}$ Northumbria Healthcare NHS Foundation Trust, Newcastle upon Tyne, UK

\subsection{6/thorax-2021-BTSabstracts.74}

Introduction The Society for Acute Medicine and the Intensive Care Society developed a collaborative evaluation of point-ofcare lung ultrasound (LUS) in the UK to describe the scope of current practice and explore performance during real-world application. All participating hospitals have established expertise in point-of-care imaging.

https://ics.ac.uk/ICS/ICS/FUSIC/Documents/National_COVID_POCUS_service_evaluation.aspx describes the project.

Methods We report the evaluation of all imaging studies performed outside the intensive care unit. An ordinal scale measured the severity of loss of lung aeration. The relationship between this score and adverse outcomes was explored using generalised linear models. A composite diagnostic score was used to describe diagnostic performance compared against polymerase chain reaction (PCR) results as a reference standard.

Results 297 ultrasound examinations from 295 patients were recorded, between February and September 2020, from 7 sites. Nasopharyngeal swab samples were positive in 145 patients (49.2\% 95\%CI 43.5-54.8). A multivariate model combining three ultrasound variables had an AUC of 0.79 (95\%CI $0.73-85)$ to predict PCR positivity. The composite outcome of death or intensive care admission at 30 days occurred in $83(28.1 \%$, 95\%CI 23.3-33.5). Lung ultrasound was able to discriminate the composite outcome with a reasonable level of accuracy (AUC 0.76 95\%CI 0.69-0.83) in univariate analysis. The relationship remained statistically 